\title{
Inhalt, Vol. 7, No. 2, 1984
}

\section{Contents}

Impressum $\quad 60$

Vorwort 62

Kleeberg, U.R., Hamburg

Geriatrische Aspekte der Onkologie 63

Fliedner, T. M. , Ulm

Gedanken zur Physiologie und Pathophysiologie des

Alterns 68

Hofmeister, G., Waiblingen

Kranksein im Alter - Überwältigung oder Bewältigung. . 85

Buchbesprechungen 88

Haag, G., Frankfurt

Entwicklungstendenzen in der Altenhilfe

Hinweise für Autoren/Sonderbände 96

Neuigkeiten für die klinische Praxis 98

Noltenius, H. und Tetzner, C, Hamburg

Vorkommen, Metastasen und natürlicher Verlauf von

behandelten und unbehandelten malignen Tumoren bei

über 70 Jahre alien Patienten 100

Erdmann, H., Hamburg

Immunkompetenz und Alter 113

Buchbesprechungen 118

Dietrich, M., Hamburg

Die malignen Hämoblastosen des älteren Menschen ... 119

Onkologie-Mitteilungen

123

Imprint 60

Preface 62

Kleeberg, U.R., Hamburg

Geriatric Oncology 63

Fliedner, T. M. , Ulm

Remarks on Physiology and Pathophysiology of Aging . . 68

Hofmeister, G., Waiblingen

Sickness in Old Age - Cope or Conquer $\quad 85$

Book Reviews 88

Haag, G., Frankfurt

Trends in Old Age Care 
Noltenius, H. and Tetzner, C, Hamburg

Occurrence, Metastasis and Natural Course of Treated

and Non-Treated Malignant Tumors in Patients older

than 70 Years 100

Erdmann, H., Hamburg

Immune System in Age

113

Book Reviews 118

Dietrich, M., Hamburg

Hematological Malignancies in the Elderly Patient . ... 119

Oncology Communications 123

Bibliographischer Hinweis: Inhaltsverzeichnisse dieser Zeitschrift erscheinen regelmäßig in current contents ${ }^{\circledR}$ sowie in anderen bibhographischen Diensten. 